Images dans le monde ibérique et ibéricoaméricain

6 | 2013

Images dans le monde ibérique et ibéro-américain

\title{
Mise en scène, modalités et stratégies du refus dans « Cartilla (poética) » de Blas de Otero
}

\section{Henry Gil}

\section{(2) OpenEdition}

Journals

Édition électronique

URL : http://journals.openedition.org/agedor/809

DOI : 10.4000/agedor.809

ISSN : 2104-3353

Éditeur

Laboratoire LISAA

Référence électronique

Henry Gil, « Mise en scène, modalités et stratégies du refus dans « Cartilla (poética) » de Blas de Otero », L'Âge d'or [En ligne], 6 | 2013, mis en ligne le 01 mars 2013, consulté le 20 avril 2019. URL : http:// journals.openedition.org/agedor/809; DOI : 10.4000/agedor.809

L'Âge d'or. Images dans le monde ibérique et ibéricoaméricain 
Henry, GIL

Université de Paris-Est Marne-la-Vallée, LISAA/EMHIS EA 4120

\title{
Mise en scène, modalités et stratégies du refus dans «Cartilla (poética) » de Blas de Otero
}

\begin{abstract}
Résumé : «Cartilla (poética) », poème métapoétique otérien, fait du concept du refus le principe générateur du texte autant d'un point de vue formel que sémantique à partir d'un débat entre deux conceptions de la poésie. Car s'il y a un refus qui, émanant d'une autorité intransigeante, impose, ici, divers commandements et par conséquent refuse d'autres orientations, il en est un autre, son contraire, qui, lui, est le refus libérateur du refus aliénant. Mais ce dernier pour déjouer la censure doit inventer ses propres modalités et stratégies en pratiquant un art du double sens et en usant d'un verbe allusif-élusif propre au « escribo y callo » otérien. C'est en créant une image filée de type allégorique qui choisit l'univers de la classe comme celui d'un pouvoir officiel qui n'accepte aucun écart et qui confère à la poésie une valeur académique, voire démiurgique, que le locuteur otérien trouve le moyen avec ironie et humour d'introduire une voix dissidente. Ici, d'un point de vue formel, tout fait sens, que ce soit le choix d'un mètre, sa disposition, l'absence d'une rime attendue ou de fortes assonances. Bien que ce poème soit un manifeste en faveur de la poésie sociale, le travail sur la langue et son conceptisme fondé sur l'usage dilogique des mots permettent d'éviter les pièges stylistiques d'un engagement social.
\end{abstract}

Mots-clés : Refus - Blas de Otero - Modalités - Stratégies - Poésie sociale - Dilogie - Verso y prosa

Resumen : "Cartilla (poética)», poema metapoético oteriano, hace del concepto de negativa el principio generador del texto tanto desde un punto de vista formal como semántico a partir de un debate entre dos concepciones de la poesía. Pues si existe una negativa que, procediendo de una autoridad intransigente, impone, aquí, diversos mandamientos y por lo tanto niega otras orientaciones, existe otra, su opuesto, que aparece como la negativa liberadora de la negativa alienante. Pero ésta para burlar la censura debe inventar sus propias modalidades y estrategias practicando un arte del doble sentido y valiéndose de un verbo alusivo-elusivo propio del « escribo y callo» oteriano. Creando una imagen continuada de tipo alegórico que escoge el ámbito de la clase como el de un poder oficial que no acepta ningún desliz y confiere a la poesía un valor académico, incluso demiúrgico, es como el locutor oteriano encuentra con ironía y humor el modo de introducir una voz disidente. Aquí, desde un punto de vista formal, todo tiene sentido, que se trate de la elección de un metro, su disposición, la ausencia de una rima esperada o de fuertes asonancias. Aunque este poema es un manifiesto a favor de la poesía social, el trabajo sobre la lengua y su conceptismo fundado en el uso dilógico de las palabras permiten evitar las trampas estilísticas de un compromiso social.

Palabras clave: Negativa - Blas de Otero - Modalidades - Estrategias - Poesía social - Dilogía Verso y prosa

À Évelyne Martin-Hernandez 


\section{CARTILLA (POÉTICA)}

La poesía tiene sus derechos.

Lo sé.

Soy el primero en sudar tinta delante del papel.

La poesía crea las palabras.

Lo sé.

Esto es verdad y sigue siéndolo diciéndola al revés.

La poesía exige ser sinceros.

Lo sé.

Le pido a Dios que me perdone y a todo dios, excúsenme.

La poesía atañe a lo esencial del ser.

No lo repitan tantas veces, repito que lo sé.

Ahora viene el pero.

La poesía tiene sus deberes. Igual que un colegial.

Entre yo y ella hay un contrato social.

Ah las palabras más maravillosas, "rosa", "poema", "mar", son $m$ pura y otras letras :

$o, a \ldots$

Si hay un alma sincera, que se guarde (en el almario) su cantar.

¿Cuántos de vida y esperanza serán?

Pero yo no he venido para ver el cielo, te advierto. Lo esencial es la existencia ; la conciencia de estar en esta clase o en la otra.

Es un deber elemental.

Blas de OTERO, Verso y prosa (1974) 


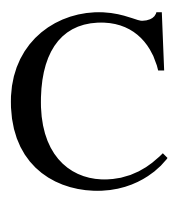

omment le concept de refus peut-il s'inscrire dans un texte poétique ? Est-il possible que ce concept soit à l'origine même du texte au point de le générer et dans ce cas quelles peuvent en être les modalités et les stratégies? Tout texte poétique étant obligé de se définir en fonction d'une tradition ou d'une histoire de la poésie en introduisant une rupture ou une relecture plus ou moins novatrice par rapport à celle-ci, ne sera-t-il pas forcément confronté à une dialectique de l'adhésion et du refus? Les réponses, on le pressent facilement, sont multiples et variées, sans oublier que dans ce genre marqué par la prosodie et par une signifiance fondée sur une indissolubilité du son et du sens, on ne peut dissocier, sans doute, plus que dans n'importe quel autre type de texte, le fond de la forme. De sorte que dans chaque poétique, la dialectique de l'adhésion et du refus doit concerner avant tout l'acceptation ou non de certaines marques formelles. J'ai choisi l'exemple particulier mais évident de Blas de Otero parce que sa voix, très tôt, s'est entièrement façonnée sur le mode du refus au sein d'une société - la société franquiste - qui était avant tout une société du refus, celui des libertés démocratiques et entre autres celle de la liberté d'expression à laquelle pouvait s'opposer le refus absolu pour l'écrivain : la censure pure et simple. On voit dès lors qu'il existe deux types de refus qui supposent un rapport conflictuel entre eux.

Il existe donc un premier refus, celui qui émane d'une autorité intransigeante, laquelle « refuse » au sens où elle « ne consent pas à accorder ce qui est demandé ». Toute demande "inconvenante », au sens ancien du terme, se voit alors "essuyer un refus ». La société franquiste au sein de laquelle écrit Blas de Otero est l'incarnation même de ce type de refus. Mais face à ce refus issu de l'autorité ou d'une figure de l'autorité - qu'il s'agisse de l'Espagne officielle, voire du Dieu sourd, insensible et invectivé en vain dans Ancia -, il est possible, par le biais du courage et de l'intelligence, de répondre par un autre refus. Un refus qui émane cette fois de l'insoumis mais qui afin de s'exprimer et de déjouer la censure devra inventer ses propres modalités et stratégies, lesquelles prendront souvent des voies détournées et un art du double sens. Le refus, dans ce cas, est donc toujours le refus du refus, autrement dit le refus libérateur du refus aliénant. "Cartilla (poética) »" le poème métapoétique au ton volontairement prosaïque, que j'ai choisi, met en scène, comme nous allons le voir, grâce à une sorte de "débat contradictoire » sur deux conceptions de la poésie, le rapport conflictuel issu de ce concept de refus dans les deux sens que je viens de rappeler et montre assez bien les objectifs et les réponses formelles propres au refus libérateur qu'instaure et revendique le poème otérien.

Le titre «Cartilla (poética)» constitue une véritable clé de lecture pour comprendre la façon dont fonctionne le poème dans la mesure où le mot «cartilla » - qui renvoie à l'abécédaire dans lequel apprenait à lire le petit écolier - introduit d'emblée un champ lexical, celui de l'école et de la classe, qui va traverser l'ensemble du poème - bien que cela soit sur un mode figuré et souvent dilogique («cartilla», «tinta», " papel», « deberes », « letras », « colegial », « clase », « deber») - et qui va créer de façon implicite une image filée à valeur allégorique où vont débattre et s'affronter deux voix, celle d'un maitre sévère,

\footnotetext{
${ }^{1}$ Ce poème apparait pour la première fois dans Que trata de España, recueil paru en 1964. Cependant nous préférons, pour notre étude, travailler sur une nouvelle version, celle de 1974, de l'anthologie Verso y prosa, composée par le poète lui-même car les très légères modifications des vers 28 et 29 (métrique pour le premier et lexicale pour le second, "Cuántos » au lieu de «Cantos ») nous semblent plus intéressantes car très riches de sens. Ce poème est choisi pour faire partie de la sous-section Obra incluse dans la section « Introducción ». Verso y prosa, edición del autor, Madrid, 22a ed., Cátedra, p. 19.
} 
intransigeant, gardien du dogme poétique et celle d'un élève rebelle, identifié au moi qui subit une autorité face à laquelle il cherche à s'émanciper. Le mot "cartilla » suggère l'expression « leerle a alguien la cartilla » c'est-à-dire « aleccionarle » ou « reprehenderle », lui faire la leçon et le réprimander. L'enjeu semble être ici celui d'une certaine conception de la poésie comme le rappelle l'adjectif "poética» dont le parenthèsage nous suggère une double lecture de ce mot, à la fois comme adjectif se rapportant à " cartilla », (un abécédaire poétique), et comme substantif ayant alors le sens d'une Poétique, autrement dit d'une «théorie sur la poésie, sur sa nature et son destin ». Cela montre d'emblée que ce poème fonctionne comme un véritable manifeste.

La structure bipartite du poème, en deux séries de quatre strophes de part et d'autre du vers 17 qui sert d'axe central, est évidente. Par ailleurs, ce monostiche semble avoir son pendant à la fin du poème avec le vers conclusif lui-même isolé. Enfin, cette structure bipartite est formellement signalée par la présence d'une rime assonante oxytonique différente dans chacune des deux séries, à chaque fois présente à la fin des vers pairs de chaque strophe - d'abord en /é/, - excepté au vers 12 où l'on trouve un mot qui ne peut rimer « excúsenme » mais nous verrons pourquoi - puis en /á/. La structure bipartite à la fois parallèle et antithétique met très clairement en évidence deux conceptions de la poésie : celle d'une "poésie pure » (v. 1-v. 16), principalement esthétisante, une poésie comme fin bien qu'elle puisse avoir également une valeur ontologique, puis, du vers 17 au vers 35, celle de la poésie sociale préconisée par le moi, fondée, en grande partie, sur le refus des dogmes énoncés auparavant. Mais ce refus est raisonné, c'est pourquoi il devient réfutation puisque chacune des quatre strophes de la seconde partie s'oppose à la strophe équivalente de la première partie : la 5 à la 1 , la 6 à la 2 , la 7 à la 3 , la 8 à la 4 .

La très grande polymétrie du poème semble être le reflet d'une discordance entre un discours académique ou officiel sur la poésie et celui du moi qui partant d'un soi-disant assentiment exprimé par le refrain «Lo sé » va glisser progressivement vers l'objection puis le refus déclaré ("Ahora viene el pero »). On pourrait même, d'ailleurs, parler ici d'un refus formel, instauré, lui, dès le début du poème. Il s'agirait alors du refus du quatrain isométrique de type hendécasyllabique, voire du cuarteto lira où alterneraient hendécasyllabes et heptasyllabes. Il n'est pas inintéressant d'observer que l'hendécasyllabe, le vers savant qui ouvre chaque strophe, est, dans la première partie, volontairement disloqué aux vers 2 et 3 en un trisyllabe suivi d'un ennéasyllabe. Il semble donc que, malgré la grande hétérométrie, on ne peut jamais parler ici de vers libres car si aucune strophe de la première partie n'utilise deux fois le même mètre, elles se construisent toutes sur une même série (hendécasyllabe/ trisyllabe/ ennéasyllabe/ heptasyllabe) $)^{2}$. Dans la deuxième partie, les deux premières strophes offrent la même hétérométrie que celles des strophes de la première partie mais dans un ordre inversé, puisqu'à l'exception de l'hendécasyllabe qui demeure toujours à l'ouverture, nous trouvons ensuite un heptasyllabe, un ennéasyllabe et un trisyllabe, comme si l'inversion, signe d'un sens contraire, entérinait formellement le refus. En revanche, les deux dernières strophes obéissent à un schéma légèrement différent, à peine retouché pour la septième strophe puisqu'elle se compose d'un hendécasyllabe, de deux ennéasyllabes et d'un trisyllabe tandis que la huitième strophe compte, elle, un

\footnotetext{
${ }^{2}$ Je ne partage pas du tout le point de vue d'Emilio Alarcos Llorach qui, malgré le mot proparoxyton à la rime (" excúsenme ») semble déplacer l'accent naturellement esdrújulo sur l'enclise afin d'obtenir un mot agudo pouvant proposer une rime oxytonique en /é/ comme dans le reste de la première partie, ce qui évidemment transforme le vers en ennéasyllabe ( $c f$. Blas de Otero, col. Clarín, Oviedo, ediciones Nobel, 1997, p. 153). Au contraire, je pense que le vers 12 se terminant par un proparoxyton est naturellement, comme tous ceux qui achèvent chaque strophe de la première partie, un heptasyllabe. En revanche, comme nous le montrerons plus loin, le refus de la rime attendue fait sens et ne pas en tenir compte appauvrit le texte.
} 
nouveau mètre, le tridécasyllabe et un vers supplémentaire puisque nous trouvons successivement un tridécasyllabe, un heptasyllabe, un ennéasyllabe, un trisyllabe et de nouveau un ennéasyllabe. Dans ces dernières strophes, les changements proviennent principalement de l'augmentation de l'ennéasyllabe, premier vers de arte mayor aussi bien savant que populaire, dont la position au sein de chaque strophe n'est jamais altérée et qui semble s'identifier ici avec ce nouveau concept qu'est la poésie sociale. On peut d'ailleurs observer que ce mètre vient clore le poème avec le second monostiche «Es un deber elemental » alors que le premier était un heptasyllabe.

Il convient de remarquer que dans la première partie, l'hendécasyllabe - vers savant par excellence - qui ouvre chaque strophe appartient à une voix extérieure et anonyme, celle de ceux qui au vers 15 apparaissent sous la forme d'une troisième personne du pluriel («No lo repitan tantas veces »). Il peut s'agir de ceux que le locuteur otérien appelle dans le poème «Y el verso se hizo hombre », «Poetas tentempié, gente ridícula» ». Or, cette voix anonyme, mais identifiée plus ou moins, comme le suggère une allégorie implicite, à celle d'un maittre d'école, énonce à chaque fois sur un mode autant conatif que référentiel une sorte de loi péremptoire, un commandement que le poète ne doit en aucun cas enfreindre. C'est ainsi que «La poesía tiene sus derechos » suggère, à travers la prosopopée de la poésie, que celle-ci ne saurait être atteinte par les maladresses ou les audaces du poèteélève, lequel vu ici comme un tâcheron revendique par l'humour et dans une forme prosaïque mais imagée, par réactivation d'un segment de discours répété ("sudar tinta») pouvant entrer dans les champs lexicaux de l'école, l'effort qu'il consacre à l'écriture ("Soy el primero en sudar tinta/ delante del papel »). En revanche, dans la strophe 5, le locuteur en reprenant l'hendécasyllabe précédent change le complément («La poesía tiene sus deberes »), puis filant la métaphore de l'école, fait endosser à la poésie le rôle d'écolier qui était auparavant le sien («Igual que un colegial») pour lui rappeler, en jouant sur la dilogie du mot « deberes », que ses devoirs, «sus tareas », sont également des obligations morales qu'elle ne peut ignorer au moment de l'écriture. Des obligations morales issues de ce que le locuteur appelle « un contrato social » entre elle et le poète. Or, ce " contrat social », qui est le fondement même de la poésie sociale, nous rappelle le titre d'un livre célèbre de JeanJacques Rousseau, Du contrat social. De sorte que par cette simple et discrète allusion à l'ouvrage de Rousseau, le locuteur en suggère implicitement le contenu : la souveraineté du peuple et l'importance d'un consentement unanime grâce auquel aucune autorité ne saurait être légitime sans le consentement de ceux qui y sont soumis. Ce refus argumenté s'exprime donc, en grande partie, de façon implicite grâce à l'art allusif-élusif de « escribo y callo $»^{4}$, propre à Otero qui, au sein d'un débat littéraire, inscrit le refus dans une dimension politique propre à la poésie sociale.

Puis face à la vision démiurgique de la poésie, née d’un second commandement («La poesía crea las palabras »), le moi, sans contester cette assertion ( Lo sé ») prétend que l'inverse est tout aussi vrai ( Esto es verdad y sigue siéndolo/ diciéndola al revés »), ce qui implique un truisme selon lequel «las palabras crean la poesía», truisme destiné à démythifier le pouvoir ésotérique ou surnaturel prêté par d'autres à la poésie. Enfin, il n'est pas impossible d'envisager que le pronom complément «la » puisse renvoyer à " poesía » dans ce cas, cela signifierait que même dite à l'envers - autrement dit dans le sens inverse à celui indiqué par le dogme - la poésie est toujours de la poésie. Cette possible lecture qui épouserait le contre-pied du dogme ne pourrait-elle être figurée par les deux premières

\footnotetext{
3 "Y el verso se hizo hombre », in Ancia, 18cima edición Madrid, Visor, p. 129.

4 Cette expression propre à l'art otérien apparaît sous forme d'épiphore dans «Biotz-Begietan », poème issu de Pido la pazy la palabra, Barcelona, ed. Lumen, octava edición 2001, pp. 53-54.
} 
strophes de la seconde partie où nous avons, comme nous l'avons constaté, un ordre inversé des mètres ? Dans la seconde partie, la strophe 6 qui est la réponse à la strophe 2, sous couvert d'émerveillement devant l'euphonie des mots employés dans les vers, dont la plupart se prêtent souvent à des topiques (« rosa», "poema», «mar»), le locuteur use d'une ironie beaucoup plus acerbe qu'auparavant envers un poème qui serait exclusivement consacré au signifiant sonore, à la musicalité du vers, au mépris d'un sens véritable. C'est ainsi qu'il emploie d'abondantes et évidentes assonances en /a/ en /o/, des allitérations en $/ \mathbf{m} /$ et des paronomases, nées de la combinaison de ces phonèmes ("MARavillosas»/ «MAR», « maravillOSAs»/ «IOSA », « oTRAS»/ «leTRAS»). Or, l'usage pléthorique du signifiant sonore dans cette strophe et le fait que ces procédés phoniques surabondants soient tous soulignés et même décodés par le locuteur lui-même non seulement leur enlèvent toute magie mais révèlent une raillerie des plus caustiques. Il en résulte donc un effet profondément comique dû, par exemple, à l'accumulation de la voyelle la plus ouverte, le /a/, employée 9 fois sur les 11 voyelles du vers 22 , ce qui force presque celui qui dit le vers - comme s'il était subitement atteint d'une béante béatitude - à se déboiter la mâchoire: («Ah 1As pAlAbrAs mÁs mArAvillosAs»). Une intention satirique du même acabit est présente avec la définition des mots employés dans le poème, comme « $m$ pura ». Car cette définition par-delà une allusion à une soi-disant pureté des graphèmes et des phonèmes ainsi qu'à un possible trope qui occupe un lieu privilégié dans le langage poétique, la metáfora pura - qui n'est autre que la métaphore in absentia - suggère forcément à un locuteur hispanophone l'euphémisme employé pour dire «mierda» comme dans l'expression «enviar o mandar a la eme ». Un euphémisme réactivé sans doute ici par le qualificatif «pura» qui suggère l'expression «pura mierda ». Ce qu'exprimerait donc ici le locuteur, à travers l'image excrémentielle, c'est le violent rejet d'une poésie où seule pourrait importer la sonorité du vers, une poésie vue alors comme une simple déjection. C'est ainsi, d'ailleurs, que le très bref vers 25 « o, a... » dénonce clairement une poésie qui se serait vidée de tout sens, dans laquelle ne subsisteraient que les supports vocaliques des interjections qui disent la fascination extatique et béate « ¡Oh! ¡Ah!».

Le troisième commandement «La poesía exige ser sinceros" ne peut que trouver l'adhésion du locuteur d'où le refrain "Lo sé », cependant celui-ci présente ses excuses comme s'il n'avait pas été ou plutôt pu être totalement sincère ( perdone »). La précision «y a todo dios excúsenme» rappelle sans équivoque, mais avec ironie, que la poésie est considérée par certains comme une sorte de dieu. Mais l'humour otérien fait que, sous couvert d'excuse et d'humilité envers cette idole, le locuteur refuse à dessein de donner au vers 12 la rime assonante oxytonique en /é/ que l'on serait en droit d'attendre. Or, l'ironie est d'autant plus forte que le mot situé à la rime, " excúsenme », qui ne peut rimer du fait que la voyelle accentuée est un /ú/, compte rien de moins que 3 /e/ atones. De sorte que l'humilité de l'énoncé, qui semble rappeler l'excuse que l'on présente après des paroles déplacées, est contredite par le refus formel de la rime attendue. Cependant, si le locuteur s'excuse devant une exigence de sincérité dont il aurait dû faire preuve en tant que poète mais qu'il n'a pu accomplir, c'est pour une raison bien précise qui ne peut être comprise que par déduction. À quoi est dû, en fait, cet aveu d'insincérité supposée ? Une insincérité toute relative, il convient de l'observer, et que l'on serait tout prêt à lui pardonner puisqu'elle est avouée. Ce que le locuteur a ici à se faire pardonner c'est ce qu'il tait. Car ce qui est à la fois tu et exprimé implicitement dans ces vers, c'est sans doute le fait de devoir passer certaines choses sous silence, autrement dit taire l'existence de la censure ou plutôt de son corollaire, l'autocensure qui oblige le poète à ne pouvoir tout dire de façon explicite sous peine d'être condamné au silence. Encore une fois le locuteur dit sans dire, il se refuse à tout dire tout en laissant entendre qu'il le devrait mais qu'il ne le 
peut. Cependant, faire entendre le refus aliénant, c'est d'une certaine façon s'en libérer, c'est le refuser en tant qu'asservissement total. Faire entendre même de façon détournée le silence imposé, ce n'est plus vraiment se taire.

De sorte que la réponse émise dans la septième strophe, comme l'a observé Emilio Alarcos Llorach, n'est pas forcément, contrairement à celle des autres strophes de cette seconde partie, une objection mais plutôt une explication ou explicitation. Mais la strophe, à vrai dire, peut s'interpréter de diverses manières. Elle joue sur l'expression « tener su alma en su almario » qui signifie ne pas être insensible à quelqu'un ou quelque chose, en particulier ici, à ceux qui dans la communauté souffrent et qui sont une majorité anonyme. Or, c'est pourtant ce que ne font pas les tenants de la première conception de la poésie, ceux qui - en quête d'une beauté formelle, voire d'une quête ontologique ou métaphysique comme le suggère la quatrième strophe - refusent que la poésie passe un «contrat social» qui devrait les obliger à se sentir solidaires du plus grand nombre, de «la inmensa mayoría ». À ceux-là, le locuteur semble leur demander, par décence, de se taire si leur chant demeure insensible au sort des autres, dans ce cas il faut lire tout simplement « Si hay un alma sincera, que se guarde su cantar» sans l'indication donnée entre parenthèses qui renvoie à l'expression idiomatique citée auparavant. En revanche, si l'on tient compte du parenthèsage nous avons l'image de "guardar su alma en su almario », image forcément polysémique car elle peut signifier soit chercher la voix de la sincérité en chantant ce qui vous touche au plus profond de vous-même, dans le tréfonds de vous-même (en el almario, o sea en la «entidad física que es soporte del alma»), soit se contenter de garder ou de ranger ce «cantar» pour l'heure au fond de vous-même, le verbe " guardar » étant amené par une paronomase implicite avec "armario », le mot littéraire "almario » apparaissant alors comme une sorte de mot-valise. Cela équivaut dans ce cas à taire, comme le fait le locuteur otérien, une partie qui ne pourra être dite telle quelle, le parenthèsage (« en el almario ») semble insister aussi sur l'idée de clôture infranchissable. Cependant l'interrogation ("¿Cuántos de vida y esperanza/serán?») - déviation parodique du célèbre titre de Rubén Darío, Cantos de vida y esperanza - qui reste sans réponse, comme pour mieux interpeller le lecteur, laisse supposer que le "cantar » peut néanmoins essayer - après s'être imprégné de la sensibilité la plus profonde, issue de l' «almario »- de devenir, s'il pratique un habile jeu allusif-élusif, un chant de vie pour survivre et d'espoir en vue d'un futur meilleur.

Le quatrième et dernier commandement qui dépasse le cadre de l'hendécasyllabe (v. 13-14), prône la valeur ontologique de la poésie («La poesía atañe a lo esencial/ del ser»). Mais cette fois-ci, le locuteur semble s'irriter profondément («No lo repitan tantas veces »). Car l'adhésion dite sous la forme de l'agacement n'est en fait que le prélude à une systématique réfutation dont nous avons vu les diverses étapes. Or, celle-ci culmine dans la dernière strophe avec le rejet pur et simple de toute poésie qui ne coïnciderait pas avec le projet marxiste d'une poésie sociale soucieuse de l'existence de la masse anonyme des hommes et de la société répartie en classes antagoniques. La strophe commencée par la conjonction adversative "Pero » exprime donc le profond rejet d'une poésie religieuse, métaphysique, contemplative, esthétisante ou simplement abstraite comme celle de l'azur mallarméen et de ses épigones "littéralistes", comme nous le fait clairement entendre le refus de lever le regard vers le ciel ("Pero yo no he venido para ver el cielo ») au lieu de le baisser vers le sol. La paronomase "cielo/suelo », ici implicite, apparaît dès la période existentielle de la voix otérienne, dans le poème "No puede» de Angel fieramente humano,

\footnotetext{
${ }^{5}$ Emilio Alarcos Llorach écrit à propos de cette troisième strophe de la « 2 da serie »: « la correspondiente estrofa de la serie segunda de reconvención no es un "pero", sino un porque », op. cit., pp. 153-154.
} 
pour suggérer déjà l'opposition entre le désir d'absolu à jamais insatisfait (el cielo) et l'existence enracinée au plus profond de la tragédie humaine (el suelo). Ce vers 30, qui dans certaines versions devient grâce à une autre préposition, un simple hendécasyllabe ("Pero yo no he venido a ver el cielo») est ici, de façon beaucoup plus pertinente, un «tridecasílabo compuesto » (7/6), sorte d'alejandrino volontairement boiteux destiné à exprimer l'imperfection humaine plus que la perfection céleste. Mais, n'oublions pas que dès «Digo vivir", le poème conclusif de Redoble de conciencia, l'existence tragique propre à l'homme est assumée et même revendiquée par le discours « agonique » du locuteur otérien avant que celui-ci ne trouve enfin avec Pido la pazy la palabra, sa raison d'être dans la solidarité avec «la inmensa mayoría », projet de la poésie sociale de Blas de Otero. À «lo esencial » fondé sur l'être en tant que «ser», but de toute quête ontologique, le locuteur oppose donc à présent « lo esencial » de l'existence qui ne peut être pour lui qu'un « estar» sociohistorique et contingent pris dans la lutte des classes et la nécessaire prise de conscience de l'appartenance à une classe sociale donnée. Pour la première fois dans le poème, il prend à témoin son lecteur en l'interpellant (" te advierto ») comme pour réduire la distance existant entre eux. Car le refus du refus est avant tout une ouverture vers l'autre, vers «son semblable, son frère » et par-delà les différences sociales qui déterminent l'existence. Enfin, dans les deux derniers vers - la fin de la dernière strophe et le monostiche conclusif - le poème s'achève sur les mots "clase » et "deber » qui sont les deux termes dilogiques qui ont permis le véritable "concepto $»^{6}$ sur lequel s'est construit par l'humour et l'ironie l'ensemble du texte. Un jeu ingénieux fondé sur la polysémie des mots et amorcé dès l'apparition du premier mot du titre, "Cartilla », donc du poème, qui a initié, comme on l'a vu, le champ lexical de l'école sur lequel se structure l'ensemble du texte.

Ce poème métapoétique portant sur deux conceptions opposées de la poésie permet, comme nous venons de le voir, de mettre véritablement en scène, grâce à un débat contradictoire, le refus. Un double refus puisque l'un émane d'un discours officiel et dogmatique, totalement inoffensif pour le pouvoir en place - lequel pour cette raison peut le protéger ou le promouvoir comme il le fit avec le garcilasismo - tandis que l'autre refus, celui du moi, s'affirme, lui, contre le premier tout en épousant certaines modalités et stratégies afin de ne pas tomber sous le coup du refus maximal qui serait la censure. Ce refus du refus est donc avant tout un acte volontaire, prémédité et fraternel qui constamment va jouer, grâce à un certain conceptisme et sur le mode de l'humour et de l'ironie - bien qu'il s'agisse de poésie sociale -, sur le double sens et l'implicite.

On voit donc que l'art suprême de ce refus réside, une fois de plus, dans le « escribo y callo » otérien, qui permet de mettre en cause non seulement une certaine conception non-engagée de la poésie mais par métonymie la société aliénante qui la sous-tend. Ce refus n'est donc pas une simple rebuffade car il se présente comme une parole engagée qui s'exprime au nom de «la inmensa mayoría » et qui tend vers une réfutation habile sans tomber pour autant dans un militantisme facile. Or, si cet écueil est évité, c'est sans doute grâce à des modalités où le sérieux n'exclut jamais l'humour ni un emploi ludique du langage bien que celui-ci, comme dans toute poésie sociale, soit utilisé comme "arma cargada de futuro » pour reprendre le mot d'ordre d'un célèbre poème de Gabriel Celaya. Si Blas de Otero est le seul de sa génération à échapper aux pièges stylistiques d'un

\footnotetext{
${ }^{6}$ Évelyne Martin-Hernández dans sa très intéressante thèse intitulée L'cuvre poétique de Blas de Otero, Université de Poitiers, dir. Alain Sicard, 1991, p. 484, avait déjà relevé la présence de ce « concepto » formé par la dilogie des mots « deber » et « clase ».
} 
engagement social qui risquerait d'appauvrir la langue et de verser dans un prosaïsme par trop facile et indigent, c'est probablement grâce au véritable et minutieux travail qu'il opère sur le langage même lorsque celui-ci semble, comme ici, spontané et proche de l'oralité. D'où un constant et subtil travail sur la signifiance et sur les mètres, dont l'objectif est d'entériner formellement le refus libérateur, comme le prouvent, entre autres, les dilogies, le refus ponctuel de la rime, et une grande polymétrie qui, malgré les apparences, est toujours significativement ordonnée. Un travail qui à cause de l'auto-vigilance infligée par la censure au poète, conduit aussi ce dernier à faire entendre, au sein même du poème, souvent par métonymie et par un art consommé de l'ellipse, un «silence assourdissant » qui n'est autre que la part émergée du refus aliénant contre lequel le locuteur lutte avec les seules armes que lui procure le langage poétique. Un langage poétique qui peut devenir, tout comme la poésie de la résistance pendant l'Occupation et selon une formule propre à René Char, « un chant du refus $»^{7}$.

\footnotetext{
${ }^{7}$ Cette expression employée par René Char dans «La poésie et la résistance », Europe, 543-544, 1974, p. 84 est reprise par Évelyne Martin-Hernández dans sa thèse à propos de Blas de Otero, op. cit., p. 259.
} 